\title{
Development of Noviomimetics as C-Terminal Hsp90 Inhibitors
}

\author{
Mercy Anyika, ${ }^{\dagger}$ Mason McMullen, ${ }^{\ddagger}$ Leah K. Forsberg, ${ }^{\dagger}$ Rick T. Dobrowsky, ${ }^{\ddagger}$ and Brian S. J. Blagg $*^{\dagger}$ \\ ${ }^{\dagger}$ Department of Medicinal Chemistry, The University of Kansas, 1251 Wescoe Hall Drive, Malott 4070, Lawrence, Kansas \\ 66045-7563, United States
}

${ }^{\ddagger}$ Department of Pharmacology and Toxicology Department, The University of Kansas, Lawrence, Kansas 66045, United States

\section{Supporting Information}

ABSTRACT: KU-32 and KU-596 are novobiocin-derived, Cterminal heat shock protein 90 (Hsp90) modulators that induce Hsp70 levels and manifest neuroprotective activity. However, the synthetically complex noviose sugar requires 10 steps to prepare, which makes translational development difficult. In this study, we developed a series of "noviomimetic" analogues of KU-596, which contain noviose surrogates that can be easily prepared, while maintaining the ability to induce Hsp70 levels. Both sugar and sugar analogues were designed, synthesized, and evaluated in a luciferase reporter assay, which identified compound 37, a benzyl containing noviomimetic, as the most potent inducer of Hsp70.

KEYWORDS: Heat Shock Protein 90, Heat Shock Protein 70, C-terminal inhibition, neuroprotection, noviomimetics

$\mathrm{H}$ eat shock protein 90 (Hsp90) is a $90 \mathrm{kDa}$ molecular chaperone that represents a promising biological target for the treatment of cancer and/or neurodegenerative diseases. It exhibits a wide range of functions stemming from its ability to assist in the folding, stability, and rematuration of proteins. Hsp90 interacts with more than 200 client proteins, many of which are oncoproteins that contribute to cancer growth and/ or resistance. ${ }^{1-6}$

There has been a tremendous focus to develop Hsp90 inhibitors for the treatment of cancer. In fact, 17 small molecules that bind the $\mathrm{N}$-terminus of $\mathrm{Hsp} 90$ have entered clinical trials. $^{7,8}$ These drugs preferentially inhibit Hsp90 and induce client protein degradation in malignant versus normal cells. $^{9-11}$ Although this selectivity aids the clinical efficacy of $\mathrm{N}$ terminal Hsp90 inhibitors, enthusiasm for their use has been dampened because they also induce the pro-survival heat shock response at the same concentration needed to inhibit client protein folding, which may limit their clinical potential. ${ }^{2}$ In contrast to $\mathrm{N}$-terminal inhibitors, we have developed novobiocin-based C-terminal inhibitors such as KU-32 and A4 (Figure 1) that can segregate induction of the heat shock response (and subsequent cytoprotection) from client protein degradation (and cytotoxicity). ${ }^{12-19}$ In fact, KU-32 was found

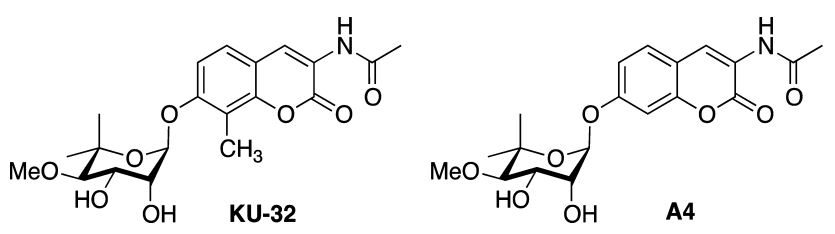

Figure 1. Chemical structures of KU-32 and A4.

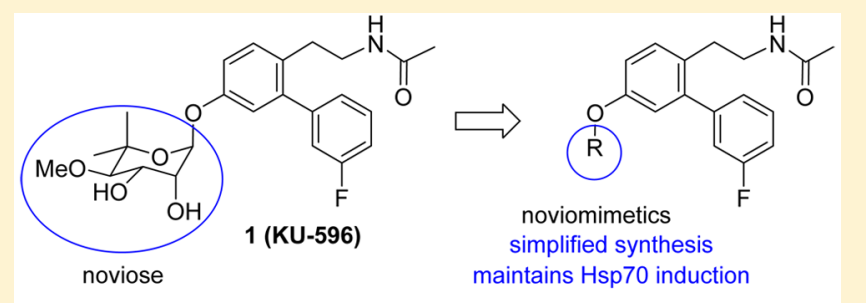

10 synthetic steps
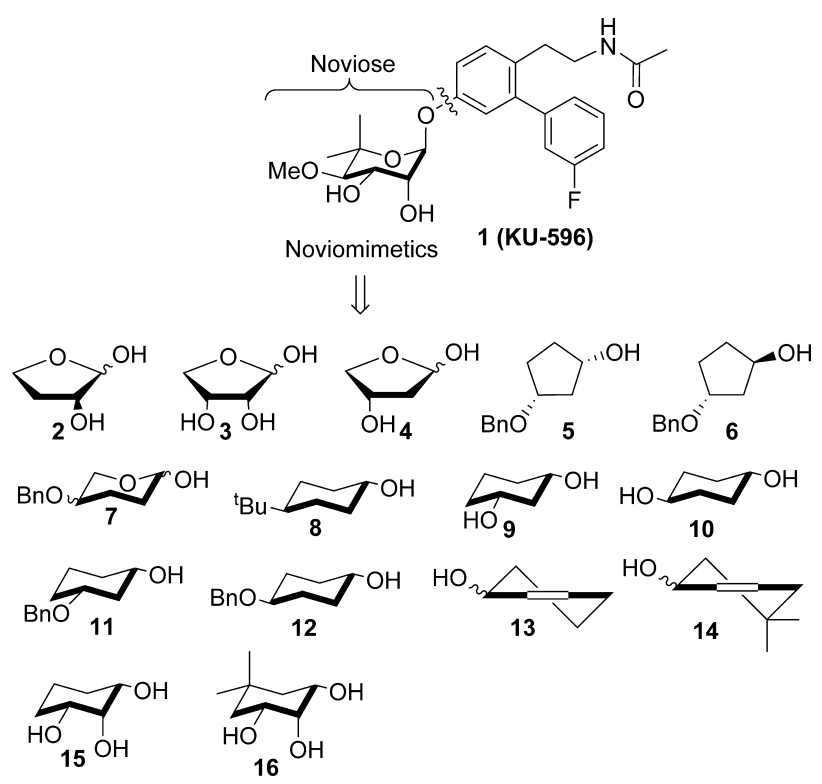

Figure 2. Structure of KU-596 (1) and sugar analogues selected for noviose replacement.

to protect against neuronal glucotoxicity and to reverse clinical end points of diabetic peripheral neuropathy in mice. ${ }^{20,21}$

In an ongoing effort to develop C-terminal Hsp90 inhibitors that manifest neuroprotective activity, KU-32 has undergone modifications to produce a second generation of noviosylated

Received: August 12, 2015

Accepted: December 7, 2015

Published: December 9, 2015 
Scheme 1. Synthesis of 5- and 6-Membered Ring Analogues

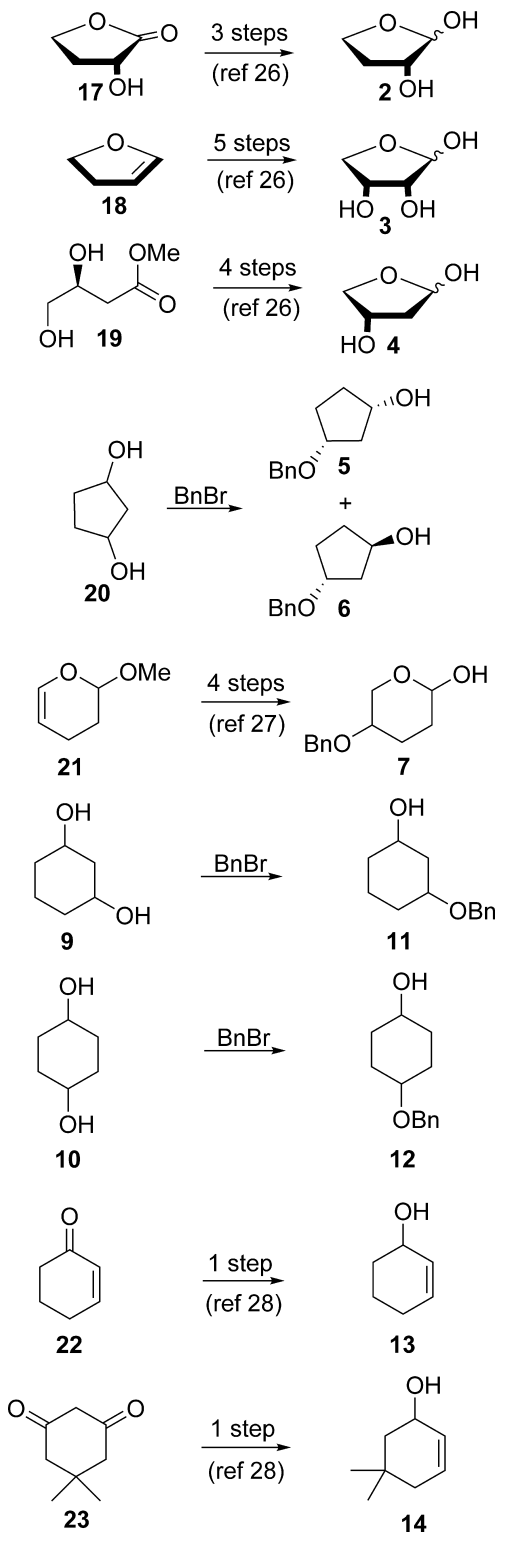

analogues, termed novologues. Novologues are small molecules that replaced the coumarin core of KU-32 with a biphenyl ring system and flexible side chain such as KU-596 (1), which manifests an enhancement in neuroprotective activity upon biological evaluation against primary sensory neurons. ${ }^{22}$ Since novologues contain noviose, a synthetically complex sugar that requires ten steps to prepare, this sugar represents a potential impediment for the translational advancement of such compounds. $^{23-25}$ To circumvent this concern, a library of succinctly prepared KU-596 analogues containing both sugar and nonsugar surrogates was generated. Previous molecular modeling studies suggested that $\mathbf{1}$ and KU-32 bind the Cterminal binding site in a manner that projects the noviose sugar into a pocket that could accommodate additional substitutions. ${ }^{22}$ Therefore, the design of noviose replacements that extend into this pocket and introduce additional interactions within the binding site were pursued. The ability of the non-noviosylated compounds to function as "noviomimetics" by promoting the induction of Hsp70 was assessed.
Scheme 2. Synthesis of Noviomimetics 25-39
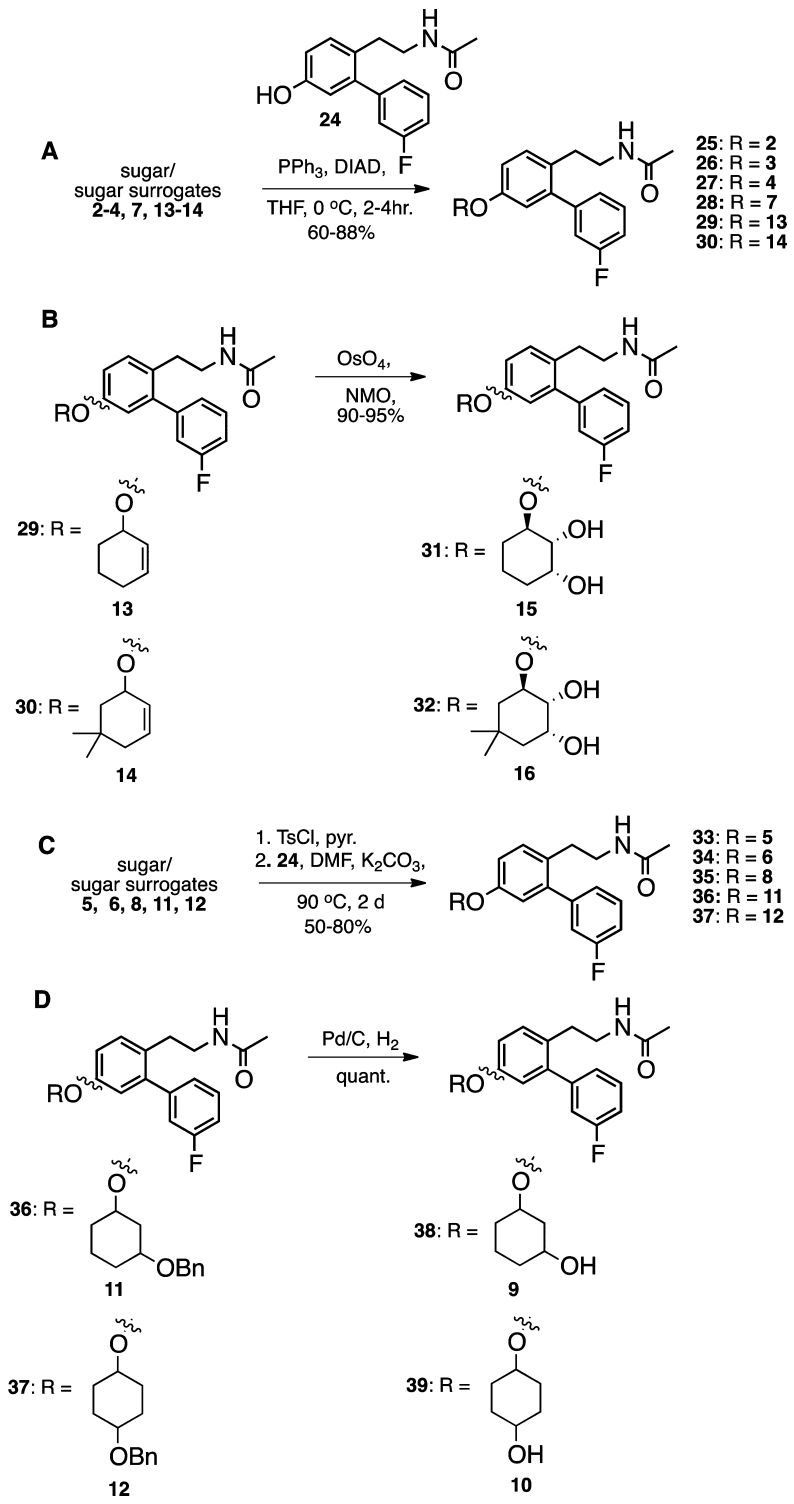

The noviose surrogates chosen for this study are shown in Figure 2 and include a series of simplified pyranoses that closely mimic the noviose chair conformation. Ring contracted furanose analogues in the envelope conformation that can project substituents into unexplored regions of the Hsp90 Cterminal binding pocket were also investigated.

As the syntheses of sugars often require many steps, simplified analogues containing a cyclohexyl or cyclopentyl ring were also investigated to determine whether a carbocyclic analogue could exhibit beneficial activity. Analogues containing an alkyl or aryl substituent were also probed to determine constraints within the binding pocket. A racemic mixture of the compounds were used in these studies.

As shown in Scheme 1, furanose derivatives 2, 3, and 4, were synthesized from commercially available $(S)-(-)$ - $\alpha$-hydroxy- $\gamma$ butyrolactone, 17, 2,3-dihydrofuran, 18, and (S)-methyl 3,4dihydroxybutanoate, 19 , respectively, via reported procedures, ${ }^{26}$ whereas cyclopentanes 5 and $\mathbf{6}$ were obtained from monobenzylation of commercially available 1,3-cyclopentadiol, 20. The resulting syn- and anti-isomers were easily separated by column chromatography. Sugar 7 was obtained from 

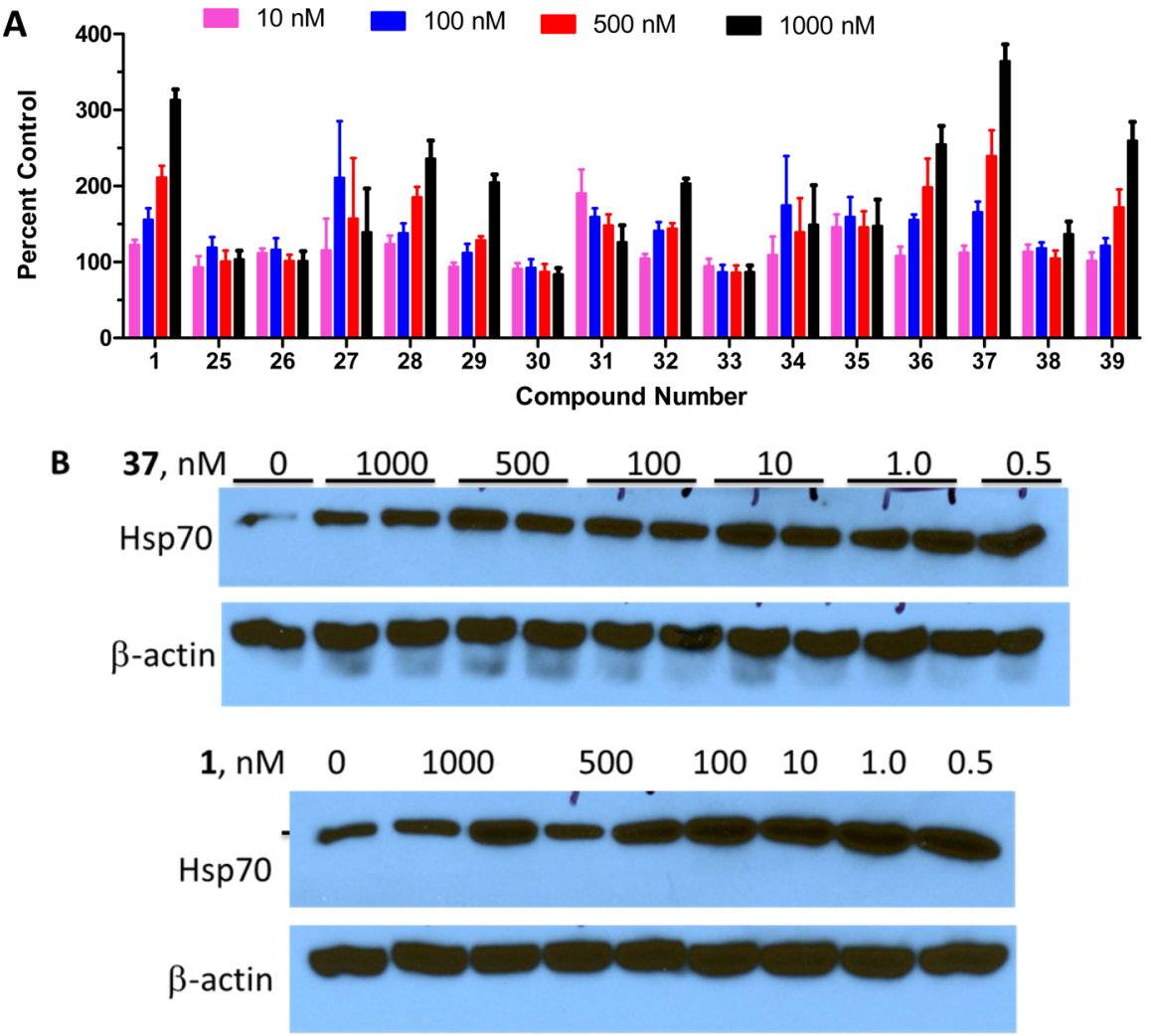

Figure 3. (A) Luciferase assay assessing Hsp70 induction by analogues 25-39. Results are mean \pm SEM ( $n=5-13)$. (B) Western Blot analysis of Hsp70 induction for 37 and 1 in nontransfected 50B11 cells.
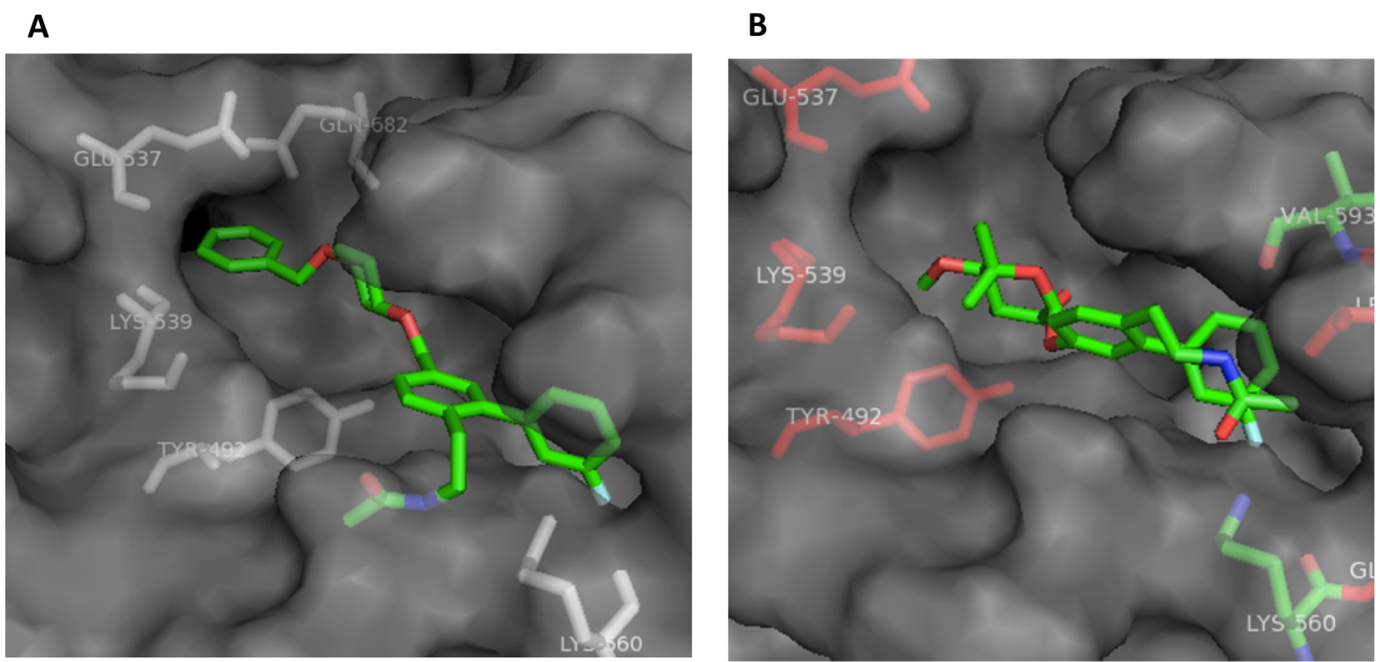

Figure 4. (A) Compound 37 docked to Hsp90 C-terminal binding site.

(B) KU-596, 1, docked to the Hsp90 C-terminal binding site.

commercially available 3,4-dihydro-2-methoxypyran, 21, via a four-step procedure. ${ }^{27}$ Cyclohexane derivatives $\mathbf{8 , 9}$, and 10 are commercially available, whereas $\mathbf{1 1}$ and $\mathbf{1 2}$ were obtained from monobenzylation of 9 and 10, respectively. Cyclohexene derivatives 13 and 14 were synthesized via published procedures from commercially available 2-cyclohexen-1-one, 22, and 5,5-dimethyl-1,3-cyclohexanedione, 23, respectively (Scheme 1). ${ }^{28}$ Aglycone $\mathbf{2 4}$ was synthesized via our previously reported procedure. ${ }^{22}$

Sugars and sugar surrogates $2,3,4,7,13$, and 14 were linked to aglycone 24 via a Mitsunobu reaction, employing triphenyl phosphine and DIAD, to obtain compounds 25-30 (Scheme
2A). Noviomimetics 29 and $\mathbf{3 0}$ were subsequently subjected to an osmium tetroxide-catalyzed dihydroxylation reaction in order to obtain the corresponding diols, 31 and 32, which contain the sugar surrogates, 15 and $\mathbf{1 6}$, respectively (Scheme 2B). To obtain compounds 33-37, sugar surrogates 5, 6, 8, 11, and 12 were first converted to the corresponding toluenesulfonates using 4-toluenesulfonyl chloride, after which they were subjected to an $\mathrm{S}_{\mathrm{N}} 2$ substitution reaction with aglycone 24 to obtain compounds 33-37 (Scheme 2C). The benzyl ethercontaining noviomimetics 36 and 37, were cleaved via hydrogenolysis to afford compounds 38 and 39, which contain the sugar surrogates, 9 and 10, respectively (Scheme 2D). 
We have previously shown that the cytoprotective activity manifested by KU-32 is dependent upon expression of Hsp70, which occurs upon Hsp90 inhibition. ${ }^{21}$ Therefore, upon construction of the library of noviomimetics, we determined their ability to induce Hsp70 via a luciferase reporter assay. In this assay, an Hsp70 promoter, which contains a heat shock binding element, is subcloned in front of a luciferase reporter gene and the resulting vector transfected into an immortalized sensory neuronal cell line (50B11 cells). The transformed cells are subsequently treated with $\mathrm{Hsp} 90$ inhibitors, which displace the transcription factor, heat shock factor 1 (HSF1), from the Hsp90 complex. Upon activation, HSF1 translocates to the nucleus, wherein it binds the Hsp70 promoter and leads to increased luciferase activity that is easily quantified. An increase in luciferase activity represents activation of the Hsp70 promoter.

Based on the results shown in Figure 3A, the derivatives that closely mimicked the chair conformation of the noviose sugar, resulted in greater Hsp70 induction. The furanose novologues $\mathbf{2 5}, \mathbf{2 6}$, and 27 were relatively inactive in the luciferase reporter assay, which suggests that the conformation manifested by the furanose derivatives does not project substituents into a favorable region of the binding pocket and, consequently, minimizes Hsp70 induction. Similarly cyclopentanes 33 and 34 did not result in significant induction. The novologues with constrained rings, $\mathbf{2 9}$ and 30, were also relatively inactive in the luciferase reporter assay, and do not mimic the chair conformation exhibited by the noviose sugar. These results collectively suggest that the novologues that mimic the chair conformation of the noviose sugar is required for $\mathrm{Hsp} 70$ induction.

Novologues that contain simplified pyranose derivatives produced varying degrees of luciferase induction. Generally, the more simplified the pyranose, the greater the Hsp70 induction. Compounds $\mathbf{3 6}$ and $\mathbf{3 7}$ were most active in the luciferase assay and represent simplified pyranose derivatives. Their nonbenzylated derivatives $\mathbf{3 8}$ and $\mathbf{3 9}$ were less active but exhibit the same trend wherein para substituents result in greater luciferase induction when compared to the meta substituent. These results suggest that not only is the chair conformation of the pyranose important for activity but larger substituents improve activity. As shown in Figure 3A, compound 37, which contains the 4-benzyl ether on the carbocyclic ring, induced the highest level of luciferase in the 50B11 transformed cellular assay and, consequently, was further investigated.

Using nontransfected 50B11 cells, compound 37 was shown to increase Hsp70 levels at concentrations similar to KU-596 (Figure 3B). In fact, 37 continued to induce a robust heat shock response even at subnanomolar concentrations.

As depicted in Figure 4A, compound 37 is suspected to bind the Hsp90 C-terminal binding site and project the aryl ring further into the pocket, which is left unoccupied in the case of KU-596, Figure 4B. Studies are currently underway to probe for additional interactions between substitutions on the aryl ring of 37 and the binding site.

In conclusion, a library of noviomimetics was designed to replace the synthetically complex noviose sugar of $\mathbf{1}$ with various sugar surrogates. It was determined that a cyclohexyl derivative containing a 4-benzyl ether (37) manifested equipotent activity as KU-596, which significantly simplifies the preparation of such compounds. Furthermore, these studies suggest that noviomimetics can successfully retain the ability to induce Hsp70, a key mechanistic feature that is associated with the neuroprotective activity manifested by the novologue class of compounds, such as $\mathbf{1}$, as well as the novobiocin-based compounds (e.g., KU-32). Thus, noviomimetics may represent a new series of synthetically simple neuroprotective compounds for the treatment of neurodegenerative diseases.

\section{ASSOCIATED CONTENT}

\section{S Supporting Information}

The Supporting Information is available free of charge on the ACS Publications website at DOI: 10.1021/acsmedchemlett.5b00331.

Preparation and evaluation of reported compounds (PDF)

\section{AUTHOR INFORMATION}

\section{Corresponding Author}

*Phone: (785) 864-2288. Fax: (785) 864-5326. E-mail: bblagg@ku.edu.

\section{Funding}

This work was supported by grants [CA109265] to B.S.J.B.; [DK095911] to R.T.D. and [NS075311] to B.S.J.B. and R.T.D. from The National Institutes of Health.

\section{Notes}

The authors declare no competing financial interest.

\section{REFERENCES}

(1) Blagg, B. S. J.; Kerr, T. A. Hsp90 inhibitors: Small molecules that transform the Hsp90 protein folding machinery into a catalyst for protein degradation. Med. Res. Rev. 2006, 26, 310-338.

(2) Zhao, H.; Michaelis, M. L.; Blagg, B. S. J. Hsp90 modulation for the treatment of Alzheimer's disease. Adv. Pharmacol. 2012, 64, 1-25.

(3) Soti, C.; Nagy, E.; Giricz, Z.; Vigh, L.; Csermely, P.; Ferdinandy, P. Heat shock proteins as emerging therapeutic targets. Br. J. Pharmacol. 2005, 146, 769-780.

(4) Chaudhury, S.; Welch, T. R.; Blagg, B. S. J. Hsp90 as a target for drug development. ChemMedChem 2006, 1, 1331-1340.

(5) Chiosis, G.; Rodina, A.; Moulick, K. Emerging Hsp90 inhibitors: From discovery to clinic. Anti-Cancer Agents Med. Chem. 2006, 6, 1-8.

(6) Peterson, L. B.; Blagg, B. S. J. To fold or not to fold: Modulation and consequences of Hsp90 inhibition. Future Med. Chem. 2009, 1, 267-283.

(7) Neckers, L.; Workman, P. Hsp90 molecular chaperone Inhibitors: Are we there yet? Clin. Cancer Res. 2012, 18, 64-76.

(8) Biamonte, M. A.; Van de Water, R.; Arndt, J. W.; Scannevin, R. H.; Perret, D.; Lee, W. Heat Shock Protein 90: Inhibitors in clinical trials. J. Med. Chem. 2010, 53, 3-17.

(9) Luo, W.; Rodina, A.; Chiosis, G. Heat shock protein 90: translation from cancer to Alzheimer's disease treatment? BMC Neurosci. 2008, 9 (Suppl 2), S7.

(10) Kamal, A.; Thao, L.; Sensintaffar, J.; Zhang, L.; Boehm, M. F.; Fritz, L. C.; Burrows, F. J. A high-affinity conformation of Hsp90 confers tumour selectivity on Hsp90 inhibitors. Nature 2003, 425, $407-410$.

(11) Chiosis, G.; Huezo, H.; Rosen, N.; Mimnaugh, E.; Whitesell, L.; Neckers, L. 17AAG: Low target binding affinity and potent cell activity - finding an explanation. Mol. Cancer Ther. 2003, 2, 123-129.

(12) Zhao, H. and Blagg, B. S. J. Inhibitors of the Hsp90 C-terminus. In Inhibitors of Molecular Chaperones As Therapeutic Agents; Machajewski, T. D., Gao, Z., Eds.; RSC Publishing: London, 2014; pp 259-301.

(13) Shelton, S. N.; Shawgo, M. E.; Matthews, S. B.; Lu, Y.; Donnelly, A. C.; Szabla, K.; Tanol, M.; Vielhauer, G. A.; Rajewski, R. A.; Matts, R. L.; Blagg, B. S. J.; Robertson, J. D. KU135, a novel novobiocin-derived C-Terminal inhibitor of the $90-\mathrm{kDa}$ heat shock protein, exerts potent 
antiproliferative effects in human leukemic cells. Mol. Pharmacol. 2009, $76,1314-1322$.

(14) Cohen, S. M.; Mukerji, R.; Samadi, A. K.; Zhang, X.; Zhao, H.; Blagg, B. S. J.; Cohen, M. Novel C-Terminal Hsp90 inhibitor for head and neck squamous cell cancer (HNSCC) with in vivo efficacy and improved toxicity profiles compared with standard agents. Ann. Surg. Oncol. 2012, 19 (Suppl 3), S483-S490.

(15) Burlison, J. A.; Avila, C.; Vielhauer, G.; Lubbers, D. J.; Holzbeierlein, J.; Blagg, B. S. J. Development of novobiocin analogues that manifest anti-proliferative activity against several cancer cell lines. J. Org. Chem. 2008, 73, 2130-2137.

(16) Burlison, J. A.; Blagg, B. S. J. Synthesis and evaluation of coumermycin Al analogues that inhibit the Hsp90 protein folding machinery. Org. Lett. 2006, 8, 4855-4858.

(17) Burlison, J. A.; Neckers, L.; Smith, A. B.; Maxwell, A.; Blagg, B. S. J. Novobiocin: Redesigning a DNA gyrase inhibitor for selective inhibition of Hsp90. J. Am. Chem. Soc. 2006, 128, 15529-15536.

(18) Donnelly, A.; Mays, J. R.; Burlison, J. A.; Nelson, J. T.; Vielhauer, G.; Holzbeierlein, J.; Blagg, B. S. J. The design, synthesis, and evaluation of coumarin ring derivatives of the novobiocin scaffold that exhibit antiproliferative activity. J. Org. Chem. 2008, 73, 89018920.

(19) Yu, X. M.; Shen, G.; Neckers, L.; Blake, H.; Holzbeierlein, J.; Cronk, B.; Blagg, B. S. J. Hsp90 inhibitors identified from a library of novobiocin analogues. J. Am. Chem. Soc. 2005, 127, 12778-12779.

(20) Lu, Y.; Ansar, S.; Michaelis, M. L.; Blagg, B. S. J. Neuroprotective activity and evaluation of Hsp90 inhibitors in an immortalized neuronal cell line. Bioorg. Med. Chem. 2009, 17, 17091715

(21) Urban, M. J.; Li, C.; Yu, C.; Lu, Y.; Krise, J. M.; McIntosh, M. P.; Rajewski, R. A.; Blagg, B. S. J.; Dobrowsky, R. T. Inhibiting heat-shock protein 90 reverses sensory hypoalgesia in diabetic mice. ASN Neuro 2010, 2, 189-199.

(22) Kusuma, B. R.; Zhang, L.; Sundstrom, T.; Peterson, L. B.; Dobrowsky, R. T.; Blagg, B. S. J. Synthesis and evaluation of novologues as C-terminal Hsp90 inhibitors with cytoprotective activity against sensory neuron glucotoxicity. J. Med. Chem. 2012, 55, 57975812.

(23) Rajesh, B. M.; Shinde, M. V.; Kannan, M.; Srinivas, G.; Iqbal, J.; Reddy, D. S. Enantiodivergent routes to (+) and (-)-novioses from (-)-pantolactone. RSC Adv. 2013, 3, 20291-20297.

(24) Matsushima, K.; Kino, J. Novel concise synthesis of ( \pm -Noviose and L-(+)-Noviose by palladium-catalyzed epoxide opening. Synthesis 2011, 2011, 1290-1294.

(25) Schmidt, B.; Hauke, S. Metathesis-based de novo synthesis of Noviose. Eur. J. Org. Chem. 2014, 2014, 1951-1960.

(26) Yu, X. M.; Han, H.; Blagg, B. S. J. Synthesis of mono- and dihydroxylated furanoses, pyranoses, and an oxepanose for the preparation of natural product analogue libraries. J. Org. Chem. 2005, 70, 5599-5605.

(27) Beaver, M. G.; Billings, S. B.; Woerpel, K. A. C-Glycosylation reactions of sulfur-substituted glycosyl donors: evidence against the role of neighboring-group participation. J. Am. Chem. Soc. 2008, 130, 2082-2086.

(28) Zhao, H.; Donnelly, A. C.; Kusuma, B. R.; Brandt, G. E. L.; Brown, D.; Rajewski, R. A.; Vielhauer, G.; Holzbeierlein, J.; Cohen, M. S.; Blagg, B. S. J. Engineering an antibiotic to fight cancer: optimization of the novobiocin scaffold to produce anti-proliferative agents. J. Med. Chem. 2011, 54, 3839-3853.

(29) Chen, W.; Mi, R.; Haughey, N.; Oz, M.; Höke, A. Immortalization and characterization of a nociceptive dorsal root ganglion sensory neuronal line. J. Peripher. Nerv. Syst. 2007, 12, 121130.

(30) Owen, L. N.; Robin, P. A. Alicyclic glycols. Part II. Derivatives of cyclohexane-1:4-diol. J. Chem. Soc. 1949, 320-326.

(31) Blagg, B. S. J.; Kusuma, B. R. Sundstrom, T. C-terminal Hsp90 inhibitors. PCT Int. Appl. 2013119985, 2013.
(32) Kim, J. D.; Han, G.; Zee, O. P.; Jung, Y. H. Deprotection of benzyl and $p$-methoxybenzyl ethers by chlorosulfonyl isocyanatesodium hydroxide. Tetrahedron Lett. 2003, 44, 733-735.

(33) Eckhardt, M.; Himmelsbach, F.; Eickelmann, P. Thomas, L. Glucopyranosyl-substituted benzyl-benzene derivatives, medicaments containing such compounds, their use and process for their manufacture. U.S. Pat. Appl. Publ. 20070049537, 2007.

(34) Matts, R. L.; Dixit, A.; Peterson, L. B.; Sun, L.; Voruganti, S.; Kalyanaraman, P.; Hartson, S. D.; Verkhivker, G. M.; Blagg, B. S. J. Elucidation of the Hsp90 C-Terminal Inhibitor Binding Site. ACS Chem. Biol. 2011, 6, 800-807. 